\title{
Relationship between hippocampal subfield volumes and memory function in adults born preterm with very low birth weight (VLBW)
}

\author{
Synne Aanes ${ }^{1 *}$, Knut J Bjuland ${ }^{2}$, Astrid M Winsnes Lærum ${ }^{3}$, Siri Weider ${ }^{4}$, Kari Anne I Evensen ${ }^{1,5-7}$, Marit Indredavik ${ }^{1}$, Ann Mari Brubakk ${ }^{1,3}$, \\ Asta Håberg ${ }^{8}$, Gro C Løhaugen ${ }^{9}$ and Jon Skranes ${ }^{1,9}$ \\ ${ }^{1}$ Department of Clinical and Molecular Medicine, Faculty of Medicine and Health Sciences, Norwegian University of Science \& Technology, Trondheim, Norway; \\ ${ }^{2}$ Department of Research, Sørlandet Hospital HF, Norway; \\ ${ }^{3}$ Children's Clinic, St. Olavs hospital, Trondheim University Hospital, Trondheim, Norway; \\ ${ }^{4}$ Department of Psychology, Faculty of Social and Educational Sciences, Norwegian University of Science \& Technology, Trondheim, Norway; \\ ${ }^{5}$ Department of Public Health and Nursing, Faculty of Medicine and Health Sciences, Norwegian University of Science \& Technology, Trondheim, Norway; \\ ${ }^{6}$ Unit for Physiotherapy Services, Trondheim Municipality, Norway \\ ${ }^{7}$ Department of Physiotherapy, Oslo Metropolitan University, Oslo, Norway \\ ${ }^{8}$ Department of Neuromedicine and Movement Science, Norwegian University of Science \& Technology, Trondheim, Norway \\ ${ }^{9}$ Department of Pediatrics, Sørlandet Hospital, Arendal, Norway
}

\begin{abstract}
Background: Being born preterm with very low birth weight (VLBW) is related to aberrant brain development. Hippocampus is a brain region particularly vulnerable to injury, for instance from consequences following preterm birth. The hippocampus is a complex structure with distinct subfields related to specific memory functions which are differently affected by neuropathological conditions. The relationship between deviations in hippocampal subfields and memory function has not been studied in adults born preterm with VLBW previously.

Aims: In this long-term follow-up study of a geographically based cohort (birth years 1986-88) of VLBW individuals and term born controls with normal birth weight, the main aim was to examine group differences in memory function and hippocampal volumes at age 26 years. We also explored hippocampal structurefunction relationships in the VLBW group.

Methods: Fifty-two VLBW and 82 control individuals aged 26 years were examined clinically of whom 44 VLBW and 70 controls had high-quality structural cerebral MRI at 3T. The participants were assessed with subtests from Wechsler Memory Scale and Cambridge Neuropsychological Test Automated Battery on verbal, visual and working memory. From the T1 weighted 3D images, hippocampal subfield volumes were estimated in FreeSurfer 6.0. In addition to group comparisons, partial correlations were performed in the VLBW group between hippocampal subfields' volumes and neonatal risk factors, and between reduced hippocampal subfields volumes and performance on the memory tests.

Results: Adults with VLBW had lower verbal, visual and working memory scores compared with term born control adults. Absolute hippocampal total and most subfield volumes were smaller in the VLBW group than in the control group, but with different findings in men and women. When adjusting for intracranial volume, left and right cornu ammonis fields and left dentate gyrus were smaller in VLBW women, while the right subiculum was smaller in VLBW men compared with control women and men, respectively. There was a negative correlation between days on mechanical ventilation and volume of the left subiculum in the VLBW group. Smaller left dentate gyrus volume was associated with lower visual memory performance in the VLBW group.
\end{abstract}

Conclusions: Adults born preterm with VLBW had lower performance on memory tests and smaller hippocampi compared with term born controls, and volume of dentate gyrus was associated with visual memory. Hippocampal subfield volumes seem to be differently affected following preterm birth in VLBW men and women.

\section{Introduction}

Being born preterm with a very low birth weight (VLBW $<1500$ gram) is related to an increased risk of aberrant brain development $[1,2]$. Even though there have been great improvements in perinatal medicine including medical treatment and care of very preterm born babies leading to increased survival and less severe brain damage, the incidence of more subtle diffuse brain injuries in grey and white matter is still high [3]. These injuries may have long-term consequences for early brain development resulting in different neuroimpairments, which include reduced general cognitive functioning [4], specific
${ }^{\star}$ Correspondence to: Synne Aanes, Department of Clinical \& Molecular Medicine, Faculty of Medicine \& Health Sciences, Norwegian University of Science \& Technology, 7489 Trondheim, Norway, E-mail: Synne.aanes@gmail.com

Key words: preterm birth, young adults, very low birth weight (VLBW), hippocampus, hippocampal subfield volumes, memory function

Received: March 16, 2020; Accepted: March 27, 2020; Published: March 31, 2020 
learning disabilities due to deficits in memory and executive functions [5-7], and attentional and mental health problems $[3,8,9]$.

Brain structures have different vulnerability to injuries, and one of the more vulnerable structures is the hippocampus [10-13]. The hippocampus is a complex, hetereogeneous structure situated bilaterally in the medial temporal lobe and responsible for normal development of learning and memory function [14-16]. The hippocampus is divided into different anatomically and functionally specialized, but highly interconnected subfields; the subiculum, presubiculum, parasubiculum, the cornu ammonis (CA) fields 1-4, and the dentate gyrus (DG) [17]. These subfields are considered to have distinct functions in short- and long-term memory with CA2/3 and CA4/DG more involved in short term memory while CA1 and subiculum more involved in retrieval and delayed episodic memory [18-21]. The CA1 and subiculum volumes have also been linked to delayed visual and verbal memory in older adults [20] and the left CA fields to verbal memory in healthy adults [21].

The hippocampus is sensitive to insults of hypoxia-ischemia [1113] which are common complications following preterm birth [22]. Further, other complications of preterm birth such as hypoglycemia, stress, and undernutrition are factors that may have negative effects on normal hippocampal development $[23,24]$.

Consequently, disturbances or damage to the developing hippocampus may contribute to neuroimpairments including memory deficits often reported in preterm born individuals $[25,26]$. We have previously reported reduced total hippocampal volumes (Bjuland, Rimol, Løhaugen, \& Skranes, 2014) which was related to reduced memory function at age 19-20 years in the present geographical cohort of young VLBW adults (Aanes, Bjuland, Skranes, \& Løhaugen, 2015). Although the role of the hippocampus in working memory is debated [27], we found a relationship between the volume of the right hippocampus and working memory ability in these VLBW young adults at age 19-20 years [6].

However, advances in MRI processing software $[28,29]$ have made it possible to study hippocampal subfields in clinical studies. Examples are studies that have investigated hippocampal subfield development from 4-22 years of age [30], the relationship to learning and memory function in healthy children [31], examined survivors of pediatric brain tumors [32] and adults with subjective memory impairment [33]. The studies mentioned above demonstrate that MRI based subfield volumetry provides the opportunity to non-invasively study relationships between specific hippocampal subfields and different memory functions. To our knowledge, hippocampal subfields have not been investigated in preterm born individuals, except for our study of school-aged VLBW children born in the early 2000s [34]. Reduced volumes of the dentate gyrus, which were positively associated with spatial working memory. was found in these VLBW children. For older cohorts of preterm born individuals, who have not experienced advanced neonatal medicine, even more pronounced abnormal structure-function relationships related to injury to individual hippocampal subfields may exist and should be investigated.

The main aim of the present study was to investigate memory function and hippocampal subfields in adults born preterm with VLBW and term born controls at age 26 years. Secondly, we wanted to examine if hippocampal subfield volumes were associated with neonatal risk factors, such as birth weight, number of days in the neonatal intensive care unit (NICU) and days on mechanical ventilation in the VLBW group. Thirdly, we wanted to investigate if there were any structure- function relationships between hippocampal subfields that were significantly reduced in the VLBW group compared to the control group and memory performance. We predicted reduced memory scores in the preterm born group. We also hypothesized that specific subfields of the hippocampus are vulnerable to injury or abnormal development after preterm birth, resulting in reduced hippocampal subfields in the VLBW group. Next, we expected an association between neonatal risk factors that may affect postnatal brain development, and hippocampal subfield volumes, and lastly; we predicted associations between reduced hippocampal subfield volumes and lower memory test performance suggesting possible brain structure-function relationships in the VLBW group.

\section{Material and methods}

\section{Participants}

The participants were part of a one of the first long-term followup studies of preterm born VLBW children where cerebral MRI was combined with multidisciplinary assessments at different ages. The study, headed by Professor Ann Mari Brubakk at the Norwegian University of Science and Technology, Trondheim, Norway consisted of three year-cohorts (born in 1986-1988) of VLBW children and term born controls examined clinically and with cerebral MRI at ages one [35], six [36], 14-15 [37-40], 19-20 [4,6,26] and 23 years of age [41,42]. Cortical thickness and white matter pathways have been reported in the same population at age 26 [43]. The study population investigated at the 26-year follow-up has previously been fully described [44] and only a brief summary is provided here.

VLBW group: Preterm born individuals with VLBW (BW $\leq 1500 \mathrm{~g})$ who were admitted to the neonatal intensive care unit (NICU) at St. Olavs hospital, Trondheim University Hospital in Norway in 19861988 were included in the cohort. Thirty-three (27\%) of the 121 VLBW neonates died in the neonatal period, two were excluded at birth due to congenital syndromes or malformations while two were excluded for follow-up due to severe cerebral palsy (CP). Consequently, 84 VLBW were eligible and invited for follow-up at 26 years of age, and 55 agreed to participate $(65 \%)$. The results from three adults with mild CP were excluded from the present study leaving 52 (26 men) participants. Of these, 51 completed the cognitive assessment and 48 consented to cerebral MRI. T1 weighted scans from four participants were excluded due to inferior image quality, leaving 44 adults ( 24 men) with MRI scans of appropriate quality for hippocampal sub-segmentation.

Control group: The control participants were recruited from a $10 \%$ random sample of term born (gestational age $>37$ completed weeks) children born in 1986-1988 with a birth weight above the $10^{\text {th }}$ percentile. The participants' mothers were enrolled in a multi-center study of 1200 multiparous women with singleton pregnancies before week 20 of pregnancy. In the original cohort, 120 newborns were included as term born controls. Two were excluded due congenital malformations, and of 118 eligible, two could not be reached for the 26year assessment. Eighty-two controls (69\%) agreed to participate, and of these 81 individuals ( 37 men) completed the cognitive assessment while 77 consented to cerebral MRI. Scans from five participants were excluded due to poor image quality leaving 72 controls ( $32 \mathrm{men}$ ) with MRI scans for hippocampal subsegmentation.

Non-participants: Of those eligible for assessment at age 26, 29 VLBW and 36 controls were lost to follow-up. In the VLBW group, participants did not differ from the non-participants with regards to birth weight, gestational age, and other neonatal variables, total IQ 
or SES. However, there were more VLBW men $(\mathrm{n}=21)$ who did not participate than VLBW women $(\mathrm{n}=8)$.

In the control group the non-participants had lower gestational age (39.3 weeks versus 39.9 weeks; $p=.019)$ and lower IQ (105 vs 91; $p$ $<.0001)$ than those who participated.

\section{Neuropsychological assessment}

The neuropsychological assessment was performed by three master students in psychology supervised by a trained neuropsychologist. All were blinded to group adherence and MRI findings. Memory function was assessed with the Logical Memory subtest from the Wechsler Memory Sale, $3^{\text {rd }}$ edition (WMS-III) [45] for verbal memory and selected scores from the computerized tests Paired Associates Learning (PAL) and Spatial Working Memory (SWM) from the Cambridge Neuropsychological Test Automated Battery (CANTAB) [46,47], assessing visual and working memory, respectively. The memory tests are described in Supplemental material. Full scale IQ scores (FSIQ) based on a full Wechsler Adult Intelligence Scale (WAIS-III) from the 19-year of age assessment [4] was used as a measure of general cognitive ability. The WAIS-III was administered during one session with a fixed subtest order by a trained neuropsychologist who was blinded to group adherence and neonatal history. The FSIQ, which is based on the results from 11 subtests, is considered the most reliable measurement of global intellectual functioning. For those who did not participate at the 19year follow-up (about $20 \%$ of participants), estimated IQ was based on the Wechsler Abbreviated Scale of Intelligence (WASI, Norwegian version) assessed at age 26 years.

\section{Neonatal variables}

Neonatal variables associated with poor long-term neurological outcome after preterm birth were recorded. These included birth weight, gestational age at birth, Apgar scores, number of days on mechanical ventilator, total days of stay in the neonatal intensive care unit (NICU) and any presence of brain pathology on neonatal ultrasonography.

\section{Socioeconomic status}

Socioeconomic status (SES) ranging from 1 (low) to 5 (high) was calculated according to Hollingshead two factor index of social position (adapted to today's categories) based on the education and occupation of one parent or the mean index of both parents [48]. Parental SES was calculated at the 14-15-year assessment, and supplemented at 1920 years for 3 participants in each group. SES scores were missing for 6 VLBW adults and 9 controls at the 26-year follow-up for whom SES scores were imputed using the mean SES score of the respective group.

\section{Magnetic resonance imaging}

Image acquisition: A 3T Siemens Skyra scanner with a 32 channel head coil was used to acquire the T1-weighted magnetization prepared rapid gradient echo (MPRAGE) sequence with repetition time (TR) = $2730 \mathrm{~ms}$, echo time $(\mathrm{TE})=3.45 \mathrm{~ms}$, inversion time $=1000 \mathrm{~ms}$, flip angle $=7^{\circ}$, voxel sizes of $1 \times 1 \times 1.33 \mathrm{~mm}^{3}$, and field of view $(\mathrm{FOV})=256 \mathrm{~mm}$. All scans were visually inspected by trained personnel and only scans with sufficient quality were used in further processing.

Hippocampal subfield segmentation: The freely available FreeSurfer image analysis suit version 6.0 (http://surfer.nmr.mgh. harvard.edu) [49-51] with the hippocampal subfield segmentation module [28] was used to process the MRI scans. The scans are first motion corrected, non-brain tissue is removed, Talairach transformation and segmentation of subcortical white matter and deep gray matter volumes are performed [51-54] before the subfield segmentation algorithm segments the hippocampus into subfields based on Bayesian inference from a high-resolution probabilistic atlas of ex vivo MRI data [28]. The module provides the following twelve hippocampal subfield labels for each hemisphere: CA1, CA2/3, CA4, the molecular layer (ML), the granule cell layer of the dentate gyrus (GC-DG), subiculum, presubiculum, parasubiculum, fimbria, the hippocampal-amygdaloid transition area (HATA), hippocampal tail, and hippocampal fissure. It also provides volume of the whole hippocampus, which includes all subfields except for the hippocampal fissure. This method shows moderate correlations with manual delineations $[55,56]$. In our study, a two-step quality control of subfield segmentations, as described in Cao et al. was performed for each individual [57]. The subfield segmentations were first manually inspected and volumes with obvious errors in location of the hippocampus or subfield assigments were excluded. This resulted in exclusion of two controls with bilateral segmentation errors and one control participant's left hippocampal subfields. Secondly, we performed outlier analyses according to Cao et al., where extreme subfield volumes were excluded. The latter step did not disclose any outliers.

To reduce the number of hippocampal subfield volumes, these were merged and regrouped into three combined subfields: the CA field, DG and Subiculum as suggested by Mueller et al. [58]. The rationale for doing this is that some of the smaller FreeSurfer subfields have shown to be less reliable when based solely on T1-weighted images as in this study $[28,59,60]$. The reclassification was done in the following way: CA field: CA1 + CA2/3 + Molecular layer + Subiculum; Dentate gyrus (DG): GC-DG + CA4; Subiculum = Presubiculum (see Figure 1. for illustration). This is a classification also more in line with other automated segmentation methods, such as the Automatic Segmentation of Hippocampal Subfields (ASHS) [29].

FreeSurfer also provides an estimated total intracranial volume (ICV) which we used for corrected volumes in the statistical analyses.

\section{Statistical analysis}

IBM SPSS Statistics version 25 was used to perform the statistical analyses. Differences in group variables were analyzed with the Student $t$ test or Mann-Whitney $U$ test depending on their distribution. Univariate general linear models were used for between-group comparisons of memory test scores and hippocampal subfield volumes. Group was added as a fixed factor and sex and age at assessment were added as covariates. For the memory tests, SES was also added as a covariate, while age was excluded as covariate when using scaled scores from the Wechsler Memory Scale. The hippocampal subfields were analyzed with and without intracranial volume as a covariate providing corrected and absolute volumes, respectively. We found a significant group $^{*}$ sex interaction for hippocampal volumes, and thus explored group differences in hippocampal volumes and memory performance for men and women separately. Effect size was calculated with Cohen's $d$. Cohen's $d \geq 0.4$ was considered a medium effect size and $d \geq 0.8$ a large effect size.

In the VLBW group, partial correlation analysis corrected for sex, intracranial volume and age at scan were used to explore associations between hippocampal volumes and continuous neonatal variables, except for days on mechanical ventilation where we used Spearman's rho. For the number of days in NICU and birth weight the degree of immaturity, i.e. gestational age at birth, was added as a covariate. Partial correlations were also performed to evaluate possible structure- 


\section{Combined subfields}
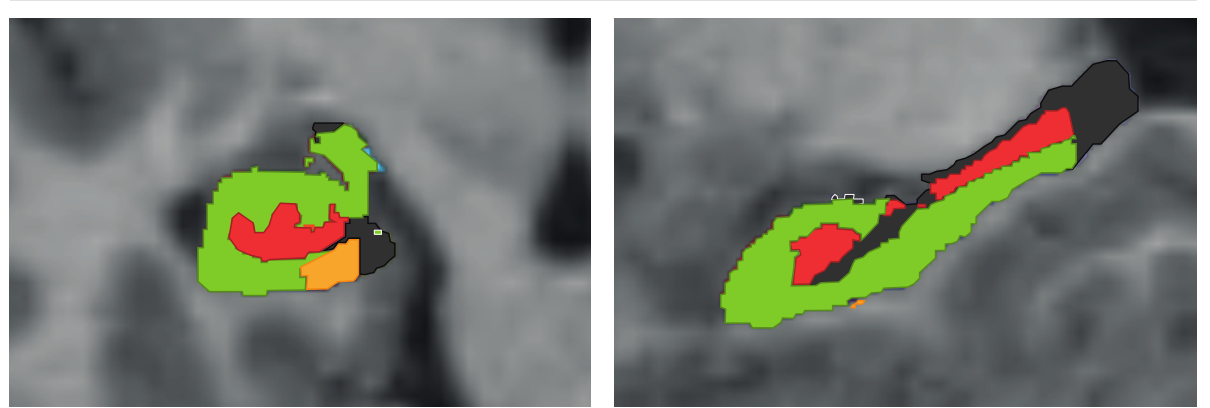

\section{Original FS6.0 subfields}
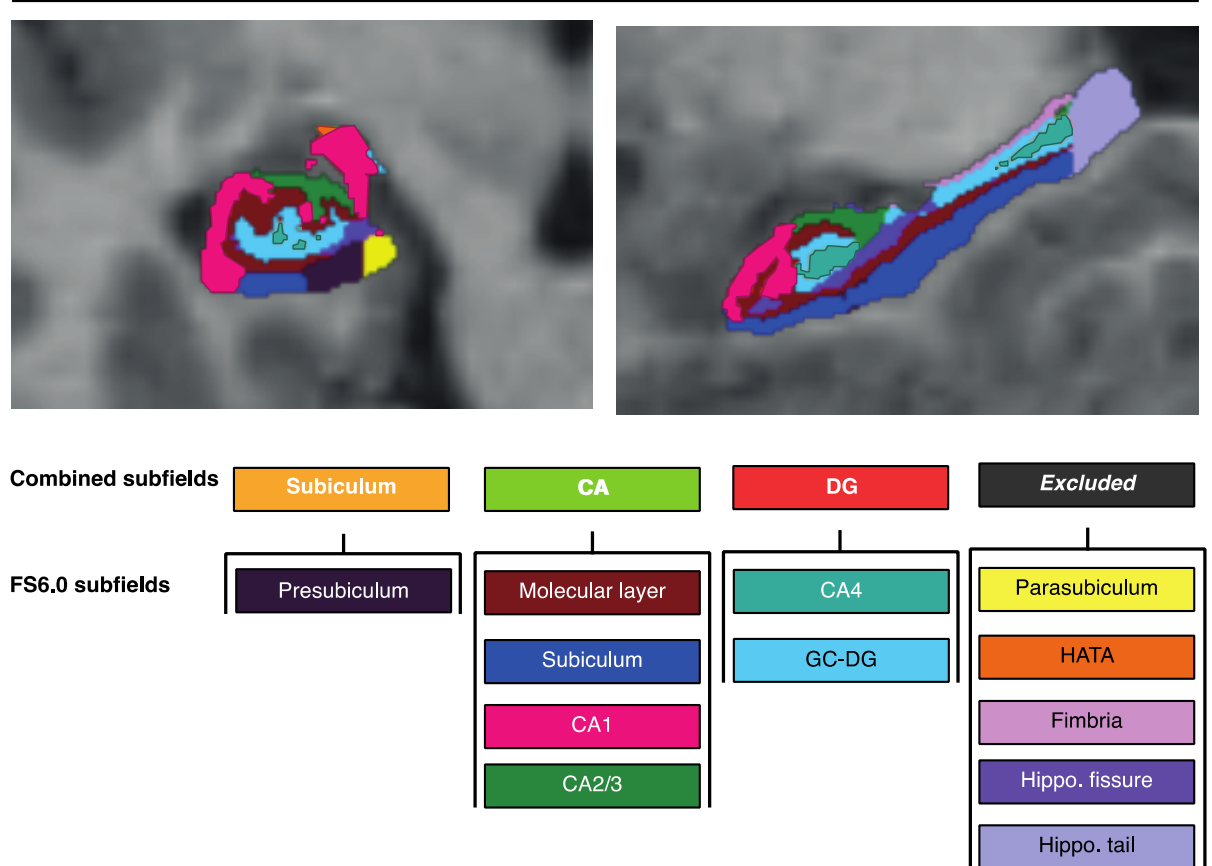

Figure 1. Combined hippocampal subfields based on original FreeSurfer (FS) 6.0 labels. The combined subfields used in the present analyses are shown in the upper row, while the original subfield labels from FreeSurfer are shown in the lower panel. Among the combined subfields the Subiculum is equal to the FreeSurfer label Presubiculum; The CA includes the FreeSurfer labels Molecular layer, Subiculum, CA1 and CA2/3; The DG consists of the FreeSurfer labels CA4 and GC-DG; while the HATA, fimbria, hippocampal fissure and hippocampal tail were all excluded from analyses

Abbreviations: $\mathrm{CA}=$ Cornu Ammonis; $\mathrm{DG}=$ Dentate gyrus; $\mathrm{GC}-\mathrm{DG}=$ Granulate cells of the dentate gyrus; HATA= Hippocampal-Amygdaloid Transition Area .

function relationships between the reduced hippocampal subfields and memory test scores in the VLBW group.

We considered two sided p-values $\leq 0.05$ as significant and corrected for multiple comparisons using the Bonferroni method. Bonferroni adjusted $\mathrm{p}$-values of significance for the group comparisons of memory test scores were $(0.05 / 4)=0.0125$, for the subfield volume analyses $(0.05 / 6)=0.0083$ and for the possible structure-function relationships $(0.05 / 16)=0.003125$. The Bonferroni corrected p-value for the correlation analyses between neonatal variables and hippocampal subfield volumes was $(0.05 / 24)=0.002$.

\section{Ethics}

The Code of Ethics of the World Medical Association (Declaration of Helsinki) was followed when conducting this study. The Regional Committee for Medical Research and Ethics approved the study protocol (project number: 2013/636/REK midt) and the participants gave written, informed consent.

\section{Results}

\section{Clinical characteristics}

Clinical group characteristics are presented in Table 1. As expected, significant differences in mean birth weight, gestational age and Apgar scores were found between the two groups. The VLBW group had mean birth weight of 1227 (SD 240) grams and gestational age of 29 (SD 2.6) weeks, while the control group had mean birth weight of 3714 (SD 438) grams and gestational age of 40 (SD 1.2) weeks. The VLBW group had lower parental SES than the control group. Thirty-one VLBW participants were in need of mechanical ventilation with a mean period of 8 days on ventilator (median 3 days). Seven VLBW participants had intraventricular hemorrhage (IVH) in the neonatal period, four with grade 1 , two with grade 2 and one with grade $4 \mathrm{IVH}$. The VLBW adults had lower mean IQ than controls (IQ score 91 vs 105, p < 0.0001), and four VLBW individuals had 
IQ scores below 70, i.e. mental retardation. There were no significant group differences regarding sex or age at assessment.

\section{Memory assessment}

The results from the memory tests for the two groups are presented in Table 2. The VLBW adults had lower immediate and delayed verbal memory measured by the logical memory test compared with controls. They also had reduced visual and spatial working memory assessed by the two CANTAB tests. When we excluded four participants with IQ $<70$ in the VLBW group, the visual memory $(\mathrm{p}=.014)$ and spatial working memory $(\mathrm{p}<.0001)$ remained significantly lower in the VLBW group. When exploring group differences based on sex, the VLBW men performed worse than men in the control group on visual memory ( $\mathrm{p}$ $=.041$ ), while both VLBW men and women performed worse than controls respectively on the spatial memory $(\mathrm{p}=.001$ for women and $\mathrm{p}$ $=.004$ for men). The group difference in verbal memory performance did not survive Bonferroni correction for multiple comparisons; however the effect sizes were moderate $(d=0.41)$ for these subtests.

\section{Hippocampal total and subfield volumes}

The VLBW adults had significantly reduced hippocampal total volumes bilaterally compared with term born controls. Also, the absolute volumes of the right and left CA field, and left DG and right subiculum were lower in the VLBW group (Supplemental material, Table S1). However, total intracranial volume was also significantly lower in the VLBW group compared to controls. After correcting for intracranial volume, total hippocampal volumes were no longer significantly reduced in the VLBW group, while the left CA field was still reduced (Table $3 \mathrm{~A}$ ), although it did not survive Bonferroni correction $(d=0.40)$.

We found a significant group versus sex interaction for several hippocampal subfields which necessitated subgroup analyses split on sex. The VLBW women had smaller total hippocampal volumes and reduced absolute volumes of CA fields and DG bilaterally compared with control women. Among men, only the absolute volume of the right subiculum was smaller in the VLBW group (Table S1). After adjusting for ICV, all group differences in hippocampal subfields, except the right DG in women, remained significant, indicating reduction in specific hippocampal subfield volumes in the VLBW group (Tables 3B \& 3C). When adjusting for multiple comparisons with Bonferroni no group differences were significant, although several subfield volumes in women had moderate effect sizes.

The absolute volumes of all the twelve hippocampal subfields segmented by FreeSurfer for the two study groups are shown in Supplemental material Table S2.

\section{Associations between neonatal variables and hippocampal subfield volumes in the VLBW group}

There was a significant negative correlation between days on mechanical ventilation and volume of the left subiculum $(r=-.427, \mathrm{p}$

Table 1. Clinical characteristics of the two study groups

\begin{tabular}{|c|c|c|c|c|c|c|c|}
\hline & \multicolumn{3}{|c|}{ VLBW group } & \multicolumn{3}{|c|}{ Control group } & \multirow[t]{2}{*}{ p-value } \\
\hline & $\mathbf{n}$ & Mean (SD) & Range & n & Mean (SD) & Range & \\
\hline Birth weight (grams) & 52 & $1227(240)$ & $645-1500$ & 82 & $3714(438)$ & $2670-5140$ & $<.0001$ \\
\hline $\begin{array}{l}\text { Gestational age (weeks) } \\
\text { Sex male } n(\%) \\
\text { Socioeconomic status (SES) }{ }^{\mathrm{a}}\end{array}$ & $\begin{array}{l}52 \\
52 \\
52\end{array}$ & $\begin{array}{c}29(2.6) \\
26(50 \%) \\
3.4(1.2)\end{array}$ & $\begin{array}{c}25-35 \\
- \\
1-5\end{array}$ & $\begin{array}{l}70 \\
70 \\
82\end{array}$ & $\begin{array}{c}40(1.2) \\
37(45 \%) \\
3.9(1.0)\end{array}$ & $\begin{array}{c}37-43 \\
- \\
2-5\end{array}$ & $\begin{array}{l}<.0001 \\
.581 \\
.006\end{array}$ \\
\hline APGAR 1 minute & 51 & $6.5(2.3)$ & $0-9$ & 77 & $8.9(0.4)$ & $7-9$ & $<.0001$ \\
\hline APGAR 5 minute & 51 & $8.3(1.4)$ & $1-10$ & 78 & $9.8(1.1)$ & $1-10$ & $<.0001$ \\
\hline Days in NICU $n$ & 51 & $73(57)$ & $23-386$ & & & & \\
\hline $\begin{array}{l}\text { Days on mechanical ventilator } n^{\mathrm{b}} \\
\text { IVH } n(\%)\end{array}$ & $\begin{array}{l}31 \\
52\end{array}$ & $\begin{array}{c}8(6) \\
7(13 \%)\end{array}$ & $\begin{array}{c}1-44 \\
-\end{array}$ & & & & \\
\hline $\begin{array}{l}\text { Age at clinical assessment (years) } \\
\text { Age at MRI (years) } \\
\text { Full scale IQ }{ }^{\mathrm{c}}\end{array}$ & $\begin{array}{l}51 \\
44 \\
52\end{array}$ & $\begin{array}{c}26.4(0.5) \\
26.3(0.7) \\
91(15)\end{array}$ & $\begin{array}{c}25.5-27.5 \\
25.2-28.4 \\
62-127\end{array}$ & $\begin{array}{l}81 \\
70 \\
82\end{array}$ & $\begin{array}{c}25.5(0.3) \\
26.4(0.4) \\
105(13)\end{array}$ & $\begin{array}{c}25.8-27.5 \\
25.6-27.3 \\
82-145\end{array}$ & $\begin{array}{c}.116 \\
.322 \\
<.0001\end{array}$ \\
\hline
\end{tabular}

Participant characteristics; Student's T-test was used for parametric data, Mann-Whitney U test for non-parametric data, Pearson's Chi-Square for categorical data. Abbreviations: VLBW = very low birth weight; NICU = neonatal intensive care unit; IVH = intraventricular hemorrhage; IQ = intelligence quotient.

aSES was calculated according to Hollingshead's two factor index of social position, based on education and occupation of one parent, or the mean index of both parents. 31 ( $60 \%$ ) of the 52 VLBW were in need of mechanical ventilation in the neonatal period. 'Full scale IQ scores from WAIS-III assessment at 19 years of age supplemented with estimated Full IQ (WASI) from 26 years for those $(20 \%)$ who did not participate at age 19 .

Table 2. Memory test results in the VLBW and control groups

\begin{tabular}{|c|c|c|c|c|c|c|}
\hline \multirow[b]{2}{*}{ Memory test } & \multicolumn{2}{|c|}{ VLBW group $(n=51)$} & \multicolumn{2}{|c|}{ Control group $(n=81)$} & \multirow[b]{2}{*}{ Cohen's $d$} & \multirow[b]{2}{*}{ p-value } \\
\hline & Mean & $95 \%$ CI & Mean & $95 \%$ CI & & \\
\hline $\begin{array}{l}\text { Verbal Memory } \\
\text { - Immediate (Logical Memory I) } \\
\text { - Delayed (Logical Memory II) }\end{array}$ & $\begin{array}{c}9.5 \\
10.4\end{array}$ & $\begin{array}{l}8.7-10.2 \\
9.6-11.1\end{array}$ & $\begin{array}{l}10.6 \\
11.5\end{array}$ & $\begin{array}{l}10.0-11.3 \\
10.9-12.1\end{array}$ & $\begin{array}{l}0.41 \\
0.41\end{array}$ & $\begin{array}{l}.019 \\
.016\end{array}$ \\
\hline $\begin{array}{l}\text { Visual Memory } \\
\text { - PAL total errors }\end{array}$ & 9.2 & $7.1-11.3$ & 5.1 & $3.7-6.5$ & 0.64 & .002 \\
\hline $\begin{array}{l}\text { Working Memory } \\
\text { - SWM errors }\end{array}$ & 29.4 & $24.0-34.7$ & 13.6 & $9.4-17.9$ & 0.87 & $<.0001$ \\
\hline
\end{tabular}

General linear model with group as fixed factor and socioeconomic status and sex as covariates for the scaled scores in verbal memory tests, and group as fixed factor and socioeconomic status, sex and age at assessment as covariates for visual and working memory tests. Cohen's $d \geq 0.4$ is indicated in bold. Abbreviations: VLBW: very low birth weight; CI: Confidence Interval; PAL = Paired Associates Learning; $\mathrm{SWM}=$ Spatial Working Memory. ${ }^{\mathrm{a}} \mathrm{n}=48 \mathrm{VLBW}, 78$ controls, ${ }^{\mathrm{b}} \mathrm{n}=49 \mathrm{VLBW}, 77 \mathrm{controls}$. 
Table 3. Hippocampal total and subfield relative volumes $\left(\mathrm{mm}^{3}\right)$ in the two study groups (3A), and stratified analyses by sex (3B and C)

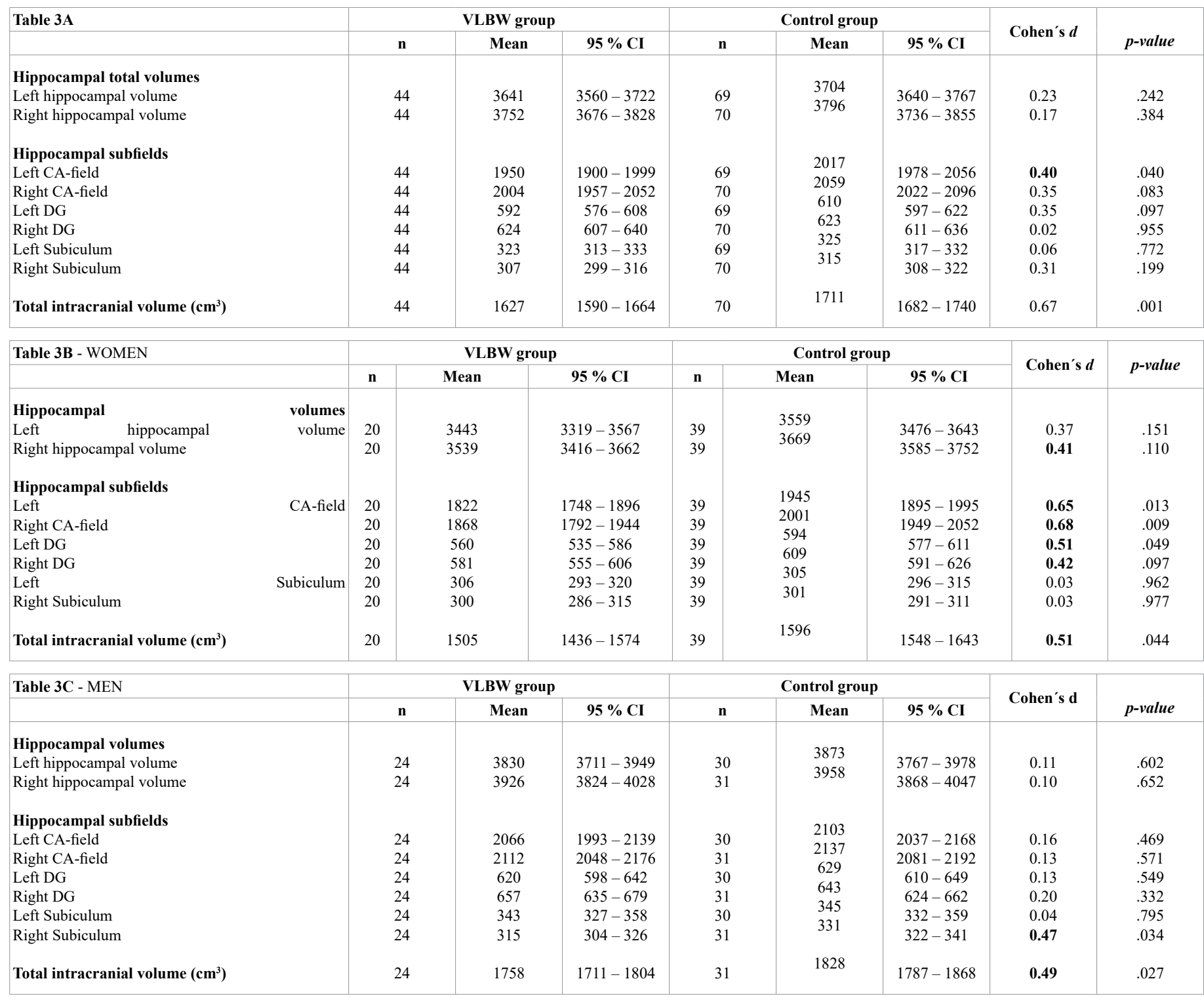

General linear model with group as fixed factor and age at MRI and intracranial volume as covariates. Cohen's $d \geq 0.4$ is indicated in bold. Abbreviations: VLBW= very low birth weight; $\mathrm{CI}=$ confidence interval; $\mathrm{CA}=\mathrm{C}$ Cornu Ammonis; $\mathrm{DG}=$ dentate gyrus.

$=.030$ ) indicating that more days on ventilator were associated with lower subicular volume in the VLBW group. This finding remained significant when removing an outlier with 35 days on ventilator $(r=$ $-.444, \mathrm{p}=.026$ ) (the participant with 44 days on ventilator mentioned in Table 1 did not have MRI). However, the correlation did not survive Bonferroni correction. There were no associations between the other neonatal variables (i.e. gestational age, birth weight and days in NICU) and hippocampal subfield volumes.

\section{Associations between reduced hippocampal subfield volumes and memory function in the VLBW group}

Volume of the left DG was negatively correlated with visual memory assessed by PAL total errors from CANTAB $(\mathrm{r}=-.374, \mathrm{p}=$ .022 ). This means that smaller volumes were associated with more errors on the visual memory test in the VLBW group. The correlation did not survive Bonferroni correction.

\section{Discussion}

The VLBW adults had reduced immediate and delayed verbal memory as well as reduced visual and spatial working memory compared with controls. Smaller absolute hippocampal total volumes and smaller absolute and relative subfield volumes of the bilateral CA fields, dentate gyrus and the right subiculum were uncovered in the VLBW adults compared with term-born adult controls. The subfield volume reductions were mainly found in VLBW women. Furthermore, there was a negative correlation between days in need of mechanical ventilation and volume of the left subiculum in the VLBW group. Reduced volume of the left dentate gyrus was associated with lower visual memory in the VLBW adults.

\section{Strength and limitations}

A major strength of this study is the prospective design where the study participants have been examined with cerebral MRI and clinical 
assessments at regular intervals from childhood. We used quantitative MRI and a rather novel and recently improved algorithm for automatic hippocampal subfield segmentation. To our knowledge, this is the first study to explore hippocampal subfield volumes in adults born preterm with VLBW. Standardized tests were used to assess different aspects of memory function.

However, there are some limitations that need to be considered. First, the rather small sample size may have resulted in too little power to detect group differences of clinical importance that did not reach statistical significance, especially after Bonferroni correction for multiple comparisons. The likelihood of type II errors (false negative) is increased by the use of the rather conservative Bonferroni method on such a sample size so that real differences between groups may appear non-significant [61]. However, presenting uncorrected p-values only may increase the risk of type I errors or false positive results. In our study uncorrected p-values revealed consistently lower volumes and performance in the VLBW group compared with controls, which are biological plausible results and probably not due to chance. This was also supported by group differences of medium effect size when using Cohen's $d$, indicating that they may be of clinical importance.

Possible selection bias with regard to participants and nonparticipants cannot be excluded in our study. Although neonatal variables did not differ in VLBW participants and non-participants, VLBW non-participating men had lower IQ than those who participated (IQ 88 versus $96, \mathrm{p}=.108$ ). Previous studies of clinical groups have shown that participants with more school problems, lower education and SES are more likely to decline participation [62-64], making it possible that individuals with the lowest performance are lost to follow-up. This could indicate that the true group differences were underestimated in our study. However, since the same trend was observed among the participating controls, i.e. they also had higher IQ than non-participants in our study, the group differences could also be driven in the opposite direction.

The automated segmentation method has some advantages compared to manual segmentation which is more laborious, less reproducible and has potential bias (Mueller et al., 2018). However, automated segmentation also has some important limitations that need consideration. Although, the method has been proven appropriate and reliable in datasets with different resolution and contrast $[28,65]$ and has shown decent correlations with manual methods (de Flores et al., 2015; Schmidt et al., 2018) as well as high test-retest reliability for all subfields except for the hippocampal fissure [65], it is recommended to use a combination of $\mathrm{T} 1$ and $\mathrm{T} 2$-weighted high resolution data. In our study, only $\mathrm{T} 1$ images were available unfortunately. The accuracy on lower resolution structural MRI data of automated methods has been questioned [66]. There is also an ongoing debate on definitions of subfields and placement of boundaries [67]. We have tried to overcome these limitations by using well-defined composite subfields that are more in line with other automated protocols such as ASHS [29] as suggested by Mueller et al. (Mueller et al., 2018), and thereby excluding smaller, less reliable subfields such as the hippocampal fissure, parasubiculum, HATA and fimbria. Nevertheless, it is a still a rather novel method and more studies are needed for validation of the subfield segmentation methods particularly in relevant clinical groups such as larger cohorts of preterm born individuals.

\section{Memory function}

We found lower verbal, visual and working memory performance in the VLBW adults compared with controls. This is in accordance with our results from assessment of these groups at 19-20 years of age [6]. At age 19, a full Wechsler Memory Scale (WMS) was performed, while at age 26 only the logical memory test for verbal memory from the WMS was performed and showed similar group differences. Visual and working memory was assessed with subtests from the automated CANTAB-test battery. The reason for this change in assessment tools was a focus on other aspects of neuropsychological functioning which were evaluated with CANTAB, leading to assessment of memory functions not being prioritized. Previous studies in the preterm population have also used the CANTAB test battery to assess spatial working memory function and found more errors and reduced memory function in preterm born children [68-70]. However, a study by Lenehan et al. (2016) which investigated if the different CANTAB tests distinguished between cognitive domains in healthy older adults found that the spatial working memory test did not load on to any of the assessed domains, including working memory. The paired associate learning (PAL) test was the only CANTAB test that strongly loaded on the memory domain and was confirmed to measure episodic memory [71]. The PAL test has been shown to be associated with bilateral hippocampal activation in a load-dependent manner, confirmed to assess object-location memory and recognized as a good test of hippocampal function and connectivity [72]. We were unable to find other studies that had used PAL from CANTAB to assess memory function in preterm born individuals; however at age 19 our VLBW adults scored lower than controls on the quite similar visual paired associate test from the Wechsler Memory Scale [6].

\section{Hippocampal volumes in adults with VLBW}

Reduced hippocampal volume in children and adolescents following very preterm birth has been reported by others [73]. We found reduced hippocampal total absolute and relative volumes in the same geographically based cohort at ages 19-20 years (Aanes et al., 2015; Bjuland et al., 2014). Although the hippocampal total absolute volumes were still smaller in the VLBW group at age 26, this group difference did not survive ICV correction. This may be explained by a slightly different group of participating adults at age 26 than earlier, since only 30 of the 44 (68 \%) VLBW adults and 38 of the $70(54 \%)$ controls aged 26 participated in the study at 19-20 years. Still, we found significant group differences in absolute and relative volumes of hippocampal subfields between the groups, with lower volumes of the CA-fields and DG in VLBW women and smaller left subiculum in VLBW men compared with controls. This relative reduction of specific hippocampal subfields may indicate increased vulnerability of these structures related to preterm birth (see section 4.5.), but the findings have to be confirmed in larger studies.

\section{Sex differences in brain and hippocampal development}

Sexual differentiation of the brain seem to occur early during brain development and is present in preterm born children scanned with MRI at 35 weeks of gestation with larger cerebral tissue volume in boys and larger folding area in girls [74]. The hippocampus is larger in men than women; however this difference disappears when correcting for total intracranial volume [75]. In addition, the development of the hippocampus follows different trajectories in boys and girls from adolescence with a continued increase in boys and a slight decrease in girls [76,77]. Sex differences are also seen in hippocampal functions, however, the mechanisms underlying the observed effects of sex are not fully understood [78]. For instance, sex differences in the DG seem to be take place early in development and this may explain the 
sex differences seen in memory function with better spatial memory abilities in men and better object memory in women [78].

The male brain seems particularly vulnerable to preterm birth with increased morbidity, mortality, neurodevelopmental-cognitive deficits and lower academic achievement and social functioning $[8,79]$. Studies indicate that the sex of very preterm born individuals influence mechanisms by which the developing brain is affected. Preterm born boys appear to be particularly vulnerable to adverse effects on white matter development and preterm born girls show strong correlations between neuro-anatomical variables and cognitive outcome [80]. Although a study on preterm born individuals found no sex differences in hippocampal asymmetry at birth [81], no studies, as far as we know, have investigated possible sex effects on hippocampal volumes in preterm born VLBW adults.

In our study, the hippocampal subfields were differently affected by VLBW in men and women with significant reduction in the volumes of CA fields and left DG among VLBW women and reduced volume of right subiculum in VLBW men. Tamnes et al. reported larger hippocampal subfield volumes in boys than girls aged 8-21 years [31]. In our study, we also found larger absolute subfield volumes in men compared to women in both groups, but these differences were no longer significant after adding ICV as a covariate (data not shown). Nevertheless, we found differences in absolute and relative volumes of several hippocampal subfields between VLBW women and control women, with far fewer group differences among men. This may be due to selection bias having lost more VLBW men with low IQ to follow-up. However, one could speculate that our finding is due to sex dependent vulnerability to preterm brain injury among VLBW men and women. Larger studies with preterm born survivors have to be performed to either confirm or reject our findings of sex-specific deviations in hippocampal subfields among VLBW individuals.

\section{Hippocampal subfields vulnerability related to preterm birth}

The hippocampal subfields have different functional roles and vulnerability to cerebral insults. The $\boldsymbol{C A}$ fields are particularly vulnerable to ischemia [10] which is a common complication of preterm birth that may cause injury to and/or harm further development, growth and maturation of white matter and subcortical structures leading to neuroimpairments in individuals born preterm with VLBW $[22,82]$. However, in our study the reduced CA field was only found in the VLBW women. There was no significant difference in neonatal risk factors between men and women in the VLBW group. The $D G$ is susceptible to injury following preterm birth since hypoglycemia $[83,84]$ and stress [85] have been reported to lead to loss of neurons through apoptosis, impaired neurogenesis and neuronal necrosis in this hippocampal subfield. We found smaller DG bilaterally in a cohort of school-aged children born preterm with VLBW in the 2000s (Aanes et al., 2019). In the present study both left and right DG were significantly smaller among VLBW women compared to control women, but only the left DG remained significantly smaller after correcting for ICV. However, when looking at effect sizes with Cohen's $d$ moderate effect sizes were found for CA fields and DG bilaterally. The subiculum is reported to be susceptible to hypoxic-ischemic episodes [86] and stress related to its role in regulation of the hypothalamicpituitary-adrenal (HPA)-axis [87]. The right subiculum was reduced in VLBW men compared with control men with an effect size of 0.47. We did find smaller right subiculum in the study of school-aged VLBW children as well, but we did not explore sex differences in that study (Aanes et al., 2019). We found a negative association between days in need of mechanical ventilation and the left subiculum in VLBW group at age 26 years, reflecting that more days on mechanical ventilation (as a sign of immaturity and/or morbidity) were associated with smaller volume of the subiculum. A relationship between days on ventilator and the volume of subiculum was also found in the school-aged VLBW children study (Aanes et al., 2019). We therefore speculate that the individuals with more immature lung function and in need of mechanical ventilation may have more episodes with hypoxia-ischemia and stress during the neonatal period, which may affect the developing hippocampus, and especially the subiculum. However, more and larger studies are needed to confirm these findings.

\section{Structure-function relationships}

We found a possible hippocampal structure-function relationship in the VLBW group where larger volume of the left DG was associated with better visual memory. Similarly, we found a positive association between the left hippocampal volume and visual memory measured in the young adults at age 19 years [6]. Similar results have been reported in patients with schizophrenia in whom the extent of impairment on the CANTAB PAL test correlated with observed lower hippocampal volume [88], and a relationship between visuospatial associative memory and DG [89].

In the cohort of school-aged VLBW children born in the 2000s, we found a positive association between spatial working memory and the right DG [34], while the working memory index was highly correlated to the right total hippocampal volume in the young VLBW adult study at age 19 [6]. The lack of a similar structure-function relationship between the spatial working memory index and hippocampal subfield volumes in the present study could have several possible explanations. It may be due to the nature of the CANTAB test used - the Spatial Working Memory, that has been reported not to load well on to the working memory index [71]. Another CANTAB test, Spatial Span, would have been more similar to the working memory tests included in our previous studies but was unfortunately not included in this study. Also, working memory is shown to be less dependent on the hippocampi in adulthood (Nosarti \& Froudist-Walsh, 2016) and it is possible that the VLBW adults at age 26 have gained a more mature working memory network that is less dependent on hippocampal structure and functioning. We argued in our paper on school-aged VLBW children that the association between spatial working memory and the right DG could be due to the spatial nature of the test and not working memory per se [34]. Nevertheless, these findings may indicate that deviations in DG following preterm birth may have consequences for memory functions even in adulthood. However, larger studies of VLBW individuals using more extensive memory test batteries have to be performed to explore further any structure-function relationship between memory function and hippocampal subfield volumes.

\section{Conclusions}

Adults born preterm with VLBW had reduced memory scores and smaller hippocampi compared with term born controls at age 26 years. When adjusting for ICV, total hippocampal volumes did not differ between groups, but left and right CA fields and left DG were smaller in VLBW women, while the right subiculum was smaller in VLBW men compared with control women and men, respectively. This may indicate sex differences in vulnerability of hippocampal subfields in early brain development following preterm birth. Reduced volume of the left DG was correlated to lower visual memory performance in 
the VLBW group, indicating a possible structure-function relationship that may be affected by preterm birth. Larger studies are needed before generalization of the findings from our study.

\section{Acknowledgement}

This study was performed at the Department of Clinical and Molecular Medicine at the Norwegian University of Science and Technology as part of the "NTNU Low Birth Weight in a Lifetime Perspective Study - NTNU LBW Life". We are grateful for the participation and cooperation of the adults in this study. We want to thank research assistant Sigrid Fenne Helgesen for recruitment of participants and organizing the examinations, and Ida Antonsen, Stine Bjøralt and Elise Haferstrøm for the cognitive assessment. We also want to thank Associate Professor Lars Morten Rimol for valuable feedback and help with the MRI statistical analyses and Clinical neuropsychologist and PhD student Rune Hatlestad Karlsen for assistance with CANTAB analysis. The Abel computer Cluster owned by the University of Oslo and the Norwegian Metacenter for Computational Science and operated by the Department of Research Computing at the University of Oslo, Center for Information and Technology (http://hpc.uio.no) was used for image processing. The study was supported by the Norwegian Research Council and the Joint Research Committee between St. Olavs hospital and Faculty of Medicine and Health Sciences, NTNU. The funding sources were not involved in any part of the study.

\section{References}

1. Nosarti C, Al-Asady MH, Frangou S, Stewart AL, Rifkin L, et al. (2002) Adolescents who were born very preterm have decreased brain volumes. Brain 125: 1616-1623.

2. Volpe JJ (2009) The encephalopathy of prematurity--brain injury and impaired brain development inextricably intertwined. Semin Pediatr Neurol 16: 167-178. [Crossref]

3. Farajdokht F, Sadigh-Eteghad S, Dehghani R, Mohaddes G, Abedi L, et al. (2017) Very low birth weight is associated with brain structure abnormalities and cognitive function impairments: A systematic review. Brain Cogn 118: 80-89. [Crossref]

4. Løhaugen GC, Gramstad A, Evensen KA, Martinussen M, Lindqvist S, et al. (2010) Cognitive profile in young adults born preterm at very low birthweight. Dev Med Child Neurol 52: 1133-1138. [Crossref]

5. Mulder H, Pitchford NJ, Marlow N (2010) Processing speed and working memory underlie academic attainment in very preterm children. Arch Dis Child Fetal Neonatal Ed 95: F267-F272.

6. Aanes S, Bjuland KJ, Skranes J, Løhaugen GCC (2015) Memory function and hippocampal volumes in preterm born very-low-birth-weight (VLBW) young adults. Neuroimage 105: 76-83. [Crossref]

7. Østgård HF, Sølsnes AE, Bjuland KJ, Rimol LM, Martinussen M, et al. (2016) Executive function relates to surface area of frontal and temporal cortex in very-lowbirth-weight late teenagers. Early Hum Dev 95: 47-53. [Crossref]

8. Hack M, Flannery DJ, Schluchter M, Cartar L, Borawski E, et al. (2002) Outcomes in young adulthood for very-low-birth-weight infants. Obstet Gynecol Surv 57: 342-344. [Crossref]

9. Indredavik MS, Vik T, Heyerdahl S, Kulseng S, Fayers P, et al. (2004) Psychiatric symptoms and disorders in adolescents with low birth weight. Arch Dis Child Fetal Neonatal Ed 89: F445-F450.

10. Walker M, Chan D, Thom M (2007) Hippocampus and Human Disease. In: Andersen P; Morris R; Amaral D; Bliss T; O'Keefe J, ed. The Hippocampus Book. 1st ed. Oxford University Press.

11. Busl KM, Greer DM (2010) Hypoxic-ischemic brain injury: Pathophysiology, neuropathology and mechanisms. NeuroRehabilitation 26: 5-13. [Crossref]

12. Nakamura Y, Nakashima T, Fukuda S, Nakashima H, Hashimoto T (1986) Hypoxicischemic brain lesions found in asphyxiating neonates. Acta Pathol Jpn 36: 551-563. [Crossref]

13. Ørbo MC, Vangberg TR, Tande PM, Anke A, Aslaksen PM (2018) Memory performance, global cerebral volumes and hippocampal subfield volumes in long-term survivors of Out-of-Hospital Cardiac Arrest. Resuscitation 126: 21-28. [Crossref]
14. Bohbot V, Allen JJB, Nadel L (2006) Memory deficits characterized by patterns of lesions to the hippocampus and parahippocampal cortex. Ann N Y Acad Sci 911: 355368.

15. Gadian DG, Aicardi J, Watkins KE, Porter DA, Mishkin M, et al. (2000) Developmental amnesia associated with early hypoxic-ischaemic injury. Brain 3: 499-507.

16. Scoville W, Milner B (2000) Loss of recent memory after bilateral hippocampal lesions. J Neurol Neurosurg Psychiatry 12: 103-113. [Crossref]

17. Duvernoy HM, Cattin F, Risold PY, Vannson JL, Gaudron M (2013) The Human Hippocampus: Functional Anatomy, Vascularization and Serial Sections with MRI, Fourth Edition. 4th ed. Berlin, Heidelberg: Springer Berlin Heidelberg.

18. Eldridge LL (2005) A dissociation of encoding and retrieval processes in the human hippocampus. $J$ Neurosci 25: 3280-3286.

19. Mueller SG, Chao LL, Berman B, Weiner MW (2011) Evidence for functional specialization of hippocampal subfields detected by MR subfield volumetry on high resolution images at 4T. Neuroimage 56: 851-857. [Crossref]

20. Zammit AR, Ezzati A, Zimmerman ME, Lipton RB, Lipton ML, et al. (2017) Roles of hippocampal subfields in verbal and visual episodic memory. Behav Brain Res 317 : 157-162. [Crossref]

21. Aslaksen PM, Bystad MK, Ørbo MC, Vangberg TR (2018) The relation of hippocampal subfield volumes to verbal episodic memory measured by the California Verbal Learning Test II in healthy adults. Behav Brain Res 351: 131-137. [Crossref]

22. Volpe JJ (2012) Neonatal encephalopathy: an inadequate term for hypoxic-ischemic encephalopathy. Ann Neurol 72: 156-166. [Crossref]

23. Schmidt-Kastner R, Freund TF (1991) Selective vulnerability of the hippocampus in brain ischemia. Neuroscience 40: 599-636. [Crossref]

24. Sizonenko SV, Borradori-Tolsa C, Vauthay DM, Lodygensky G, Lazeyras F, et al. (2006) Impact of intrauterine growth restriction and glucocorticoids on brain development: Insights using advanced magnetic resonance imaging. Mol Cell Endocrinol 254-255: 163-171. [Crossref]

25. Jacob FD, Habas PA, Kim K, Corbett-Detig J, Xu D, et al. (2011) Fetal hippocampal development: Analysis by magnetic resonance imaging volumetry. Pediatr Res 69: 425-429. [Crossref]

26. Bjuland KJ, Rimol LM, Løhaugen GCC, Skranes J (2014) Brain volumes and cognitive function in very-low-birth-weight (VLBW) young adults. Eur J Paediatr Neurol 18: 578-590. [Crossref]

27. Baddeley A, Jarrold C, Vargha-khadem F (2011) Working memory and the hippocampus. J Cogn Neurosci 23: 3855-3861. [Crossref]

28. Iglesias JE, Augustinack JC, Nguyen K, Player CM, Player A, et al. (2015) A computational atlas of the hippocampal formation using ex vivo, ultra-high resolution MRI: Application to adaptive segmentation of in vivo MRI, for the Alzheimer's Disease Neuroimaging Initiative 1. Neuroimage 115: 117-137. [Crossref]

29. Yushkevich PA, Pluta JB, Wang H, Xie L, Ding SL, et al. (2015) Automated volumetry and regional thickness analysis of hippocampal subfields and medial temporal cortical structures in mild cognitive impairment. Hum Brain Mapp 36: 258-287. [Crossref]

30. Krogsrud SK, Tamnes CK, Fjell AM, Amlien I, Grydeland H, et al. (2014) Development of hippocampal subfield volumes from 4 to 22 years. Hum Brain Mapp 35: 5646-5657. [Crossref]

31. Tamnes CK, Walhovd KB, Engvig A, Grydeland H, Krogsrud SK, et al. (2014) Regional hippocampal volumes and development predict learning and memory. Dev Neurosci 36: 161-174. [Crossref]

32. Decker AL, Szulc KU, Bouffet E, Laughlin S, Chakravarty MM, et al. (2017) Smaller hippocampal subfield volumes predict verbal associative memory in pediatric brain tumor survivors. Hippocampus 27: 1140-1154. [Crossref]

33. Engvig A, Fjell AM, Westlye LT, Skaane NV, Sundseth Ø, et al. (2012) Hippocampal subfield volumes correlate with memory training benefit in subjective memory impairment. Neuroimage 61: 188-194. [Crossref]

34. Aanes S, Bjuland KJ, Sripada K, Sølsnesa AE, Grunewaldt KH, et al. (2019) Reduced hippocampal subfield volumes and memory function in school-aged children born preterm with very low birthweight (VLBW). NeuroImage Clin 101857.

35. Skranes JS, Nilsen G, Smevik O, Vik T, Rinck P, et al. (1992) Cerebral magnetic resonance imaging (MRI) of very low birth weight infants at one year of corrected age. Pediatr Radiol 22: 406-409. [Crossref] 
36. Skranes JS, Nilsen G, Smevik O, Vik T, Brubakk M (1998) Cerebral MRI of very low birth weight children at 6 years of age compared with the findings at 1 year. Pediatr Radiol 28: 471-475. [Crossref]

37. Martinussen M, Fischl B, Larsson HB, Skranes J, Kulseng S, et al. (2005) Cerebral cortex thickness in 15-year-old adolescents with low birth weight measured by an automated MRI-based method. Brain 128(Pt 11): 2588-2596.

38. Skranes J, Evensen KI, Løhaugen GC, Martinussen M, Kulseng S, et al. (2008) Abnormal cerebral MRI findings and neuroimpairments in very low birth weight (VLBW) adolescents. Eur J Paediatr Neurol 12: 273-283. [Crossref]

39. Evensen KA, Vik T, Helbostad J, Indredavik MS, Kulseng S, et al. (2004) Motor skills in adolescents with low birth weight. Arch Dis Child Fetal Neonatal Ed 89: F451-455.

40. Indredavik MS, Skranes JS, Vik T, Heyerdahl S, Romundstad P, et al. (2005) Lowbirth-weight adolescents: psychiatric symptoms and cerebral MRI abnormalities. Pediatr Neurol 33: 259-266. [Crossref]

41. Husby IM, Skranes J, Olsen A, Brubakk AM, Evensen KAI (2013) Motor skills at 23 years of age in young adults born preterm with very low birth weight. Early Hum Dev 89: 747-754. [Crossref]

42. Hollund IMH, Olsen A, Skranes J, Brubakk AM, Håberg, AK, et al. (2018) White matter alterations and their associations with motor function in young adults born preterm with very low birth weight. NeuroImage Clin 17: 241-250. [Crossref]

43. Rimol LM, Botellero VL, Bjuland KJ, Løhaugen GCC, Lydersen S, et al. (2019) Reduced white matter fractional anisotropy mediates cortical thickening in adults born preterm with very low birthweight. Neuroimage 188: 217-227. [Crossref]

44. Lærum AM, Reitan SK, Evensen KA, Lydersen S, Brubakk AM, et al. (2017) Psychiatric disorders and general functioning in low birth weight adults: a longitudinal study. Pediatrics 139: e20162135. [Crossref]

45. Wechsler D (1997) Wechsler Memory Scale, third edition.

46. Cambridge Cognition (2006) CANTABeclipseTM: Version 3: Software User Guide. Cambridge, UK: Author.

47. Cambridge Cognition (2006) CANTABeclipseTM Version 3: Test Administration Guide. Cambridge, UK: Author.

48. Hollingshead A. Two factor index of social position. 1957.

49. Dale A, Fischl B, Sereno MI (1999) Cortical surface-based analysis. I. Segmentation and surface reconstruction. Neuroimage 9: 179-194. [Crossref]

50. Fischl B, Salat DH, Busa E, Albert M, Dieterich M, et al. (2002) Whole brain segmentation: automated labeling of neuroanatomical structures in the human brain. Neuron 33: 341-355. [Crossref]

51. Fischl B, van der Kouwe A, Destrieux C, Halgren E, Ségonne F, et al. (2004) Automatically parcellating the human cerebral cortex. Cereb Cortex 14: 11-22. [Crossref]

52. Kuperberg GR, Broome MR, McGuire PK, David AS, Eddy M, et al. (2003) Regionally localized thinning of the cerebral cortex in schizophrenia. Arch Gen Psychiatry 60: 878-888. [Crossref]

53. Reuter M, Rosas HD, Fischl B (2010) Highly accurate inverse consistent registration: a robust approach. Neuroimage 53: 1181-1196. [Crossref]

54. Ségonne F, Dale AM, Busa E, Glessner M, Salat D, et al. (2004) A hybrid approach to the skull stripping problem in MRI. Neuroimage 22: 1060-1075. [Crossref]

55. de Flores R, La Joie R, Chételat G (2015) Structural imaging of hippocampal subfields in healthy aging and Alzheimer's disease. Neuroscience 309: 29-50. [Crossref]

56. Schmidt MF, Storrs JM, Freeman KB, Jack CR Jr, Turner ST, et al. (2018) A comparison of manual tracing and FreeSurfer for estimating hippocampal volume over the adult lifespan. Hum Brain Mapp 39: 2500-2513. [Crossref]

57. Cao B, Passos IC, Mwangi B, Amaral-Silva H, Tannous J, et al. (2017) Hippocampal subfield volumes in mood disorders. Mol Psychiatry 22: 1352-1358. [Crossref]

58. Mueller SG, Yushkevich PA, Das S, Wang L, Van Leemput K, et al. (2018) Systematic comparison of different techniques to measure hippocampal subfield volumes in ADNI2. Neuroimage Clin 17:1006-1018. [Crossref]

59. Chen LW, Sun D, Davis SL, Haswell CC, Dennis EL, et al. (2018) Smaller hippocampa CA1 subfield volume in posttraumatic stress disorder. Depress Anxiety 35: 1018-1029. [Crossref]

60. McHugo M, Talati P, Woodward ND, Armstrong K, Blackford JU, et al. (2018) Regionally specific volume deficits along the hippocampal long axis in early and chronic psychosis. NeuroImage Clin 20: 1106-1114. [Crossref]
61. Perneger TV (1998) What's wrong with Bonferroni adjustments. BMJ 316: 1236-1238. [Crossref]

62. Krellman JW, Kolakowsky-Hayner SA, Spielman L, Dijkers M, Hammond FM, et al. (2014) Predictors of follow-up completeness in longitudinal research on traumatic brain injury: findings from the National Institute on Disability and Rehabilitation Research traumatic brain injury model systems program. Arch Phys Med Rehabil 95: 633-641. [Crossref]

63. Kristman V, Manno M, Côté P (2004) Loss to follow-up in cohort studies: how much is too much? Eur J Epidemiol 19: 751-760. [Crossref]

64. Winding TN, Andersen JH, Labriola M, Nohr EA (2014) Initial non-participation and loss to follow-up in a Danish youth cohort: implications for relative risk estimates. $J$ Epidemiol Community Health 68: 137-144.

65. Whelan CD, Hibar DP, van Velzen LS, Zannas AS, Carrillo-Roa T, et al. (2016) Heritability and reliability of automatically segmented human hippocampal formation subregions. Neuroimage 128: 125-137. [Crossref]

66. Wisse LEM, Biessels GJ, Geerlings MI (2014) A Critical Appraisal of the hippocampal subfield segmentation package in FreeSurfer. Front Aging Neurosci 6: 261. [Crossref]

67. Wisse LEM, Daugherty AM, Olsen RK, Berron D, Carr VA, et al. (2017) A harmonized segmentation protocol for hippocampal and parahippocampal subregions: Why do we need one and what are the key goals? Hippocampus 27: 3-11. [Crossref]

68. Luciana M, Lindeke L, Georgieff M, Mills M, Nelson CA (1999) Neurobehavioral evidence for working-memory deficits in school-aged children with histories of prematurity. Dev Med Child Neurol 41: 521-533.

69. Curtis WJ (2002) Neurobehavioural functioning in neonatal intensive care unit graduates in late childhood and early adolescence. Brain 125: 1646-1659. [Crossref]

70. Fitzpatrick A, Carter J, Quigley MA (2016) Association of gestational age with verbal ability and spatial working memory at age 11. Pediatrics 138: e20160578. [Crossref]

71. Lenehan ME, Summers MJ, Saunders NL, Summers JJ, Vickers JC (2016) Does the Cambridge Automated Neuropsychological Test Battery (CANTAB) distinguish between cognitive domains in healthy older adults? Assessment 23: 163-172. [Crossref]

72. Barnett JH, Blackwell AD, Sahakian BJ, Robbins TW (2016) The paired associates learning (PAL) test: 30 years of CANTAB translational neuroscience from laboratory to bedside in dementia research. Curr Topics Behavioral Neurosci 28: 449-474.

73. Nosarti C, Froudist-Walsh S (2016) Alterations in development of hippocampal and cortical memory mechanisms following very preterm birth. Dev Med Child Neurol 58: 35-45. [Crossref]

74. Vasileiadis GT, Thompson RT, Han VKM, Gelman N (2009) Females follow a more "Compact" early human brain development model than males. A case-control study of preterm neonates. Pediatr Res 66: 551-554. [Crossref]

75. Tan A, Ma W, Vira A, Marwha D, Eliot L (2016) The human hippocampus is not sexually-dimorphic: Meta-analysis of structural MRI volumes. Neuroimage 124: 350366. [Crossref]

76. Bramen JE, Hranilovich JA, Dahl RE, Forbes EE, Chen J, et al. (2011) Puberty influences medial temporal lobe and cortical gray matter maturation differently in boys than girls matched for sexual maturity. Cereb Cortex 21: 636-646. [Crossref]

77. Goddings AL, Mills KL, Clasen LS, Giedd JN, Viner RM, et al. (2014) The influence of puberty on subcortical brain development. Neuroimage 88: 242-251. [Crossref]

78. Koss WA, Frick KM (2017) Sex differences in hippocampal function. $J$ Neurosci Res 95: 539-562. [Crossref]

79. Hindmarsh GJ, O’Callaghan MJ, Mohay HA, Rogers YM (2000) Gender differences in cognitive abilities at 2 years in ELBW infants. Early Hum Dev 60: 115-122. [Crossref]

80. Reiss AL, Kesler SR, Vohr B, Duncan CC (2004) Sex differences in cerebral volumes of 8-year-olds born preterm. J Pediatr 145: 242-249.

81. Thompson DK, Wood SJ, Doyle LW, Warfield SK, Egan GF, et al. (2009) MRdetermined hippocampal asymmetry in full-term and preterm neonates. Hippocampus 19: 118-123. [Crossref]

82. Volpe JJ (2009) Brain injury in premature infants: a complex amalgam of destructive and developmental disturbances. Lancet Neurol 8: 110-124. [Crossref]

83. Aure R (1986) Progress review: hypoglycemic brain damage. Stroke 17: 699. [Crossref]

84. Auer RN (2010) Hypoglycemic brain damage. Acute neuronal inj role excit program cell death. Mech 146: 203-210. 
Aanes S (2020) Relationship between hippocampal subfield volumes and memory function in adults born preterm with very low birth weight (VLBW)

85. Morris R. Stress and the Hippocampus. In: Anderson P; Morris R; Amaral D; O' Keefe J; Bliss T, ed. The Hippocampus Book. 1st ed. Oxford University Press; 2007:751-768.

86. Stark C. Functional Role of the Human Hippocampus. In: Andersen P; Morris R; Amaral D; Bliss T; O'Keefe J, ed. The Hippocampus Book. 1st ed. Oxford University Press; 2007:549-580.

87. Herman JP, Mueller NK (2006) Role of the ventral subiculum in stress integration. Behav Brain Res 174: 215-224.
88. Kéri S, Szamosi A, Benedek G, Kelemen O (2012) How does the hippocampal formation mediate memory for stimuli processed by the magnocellular and parvocellular visual pathways? Evidence from the comparison of schizophrenia and amnestic mild cognitive impairment (aMCI). Neuropsychologia 50: 3193-3199. [Crossref]

89. Wannan CMJ, Cropley VL, Chakravarty MM, Van Rheenen TE, Mancuso S, et al. (2019) Hippocampal subfields and visuospatial associative memory across stages of schizophrenia-spectrum disorder. Psychol Med 49: 2452-2462. [Crossref]

Copyright: $@ 2020$ Aanes S. This is an open-access article distributed under the terms of the Creative Commons Attribution License, which permits unrestricted use, distribution, and reproduction in any medium, provided the original author and source are credited. 